M. I. TORO

\title{
Valores del perfil lipídico ¿Todos con el mismo rasero?
}

\section{Lipid profile values \\ All with the same standard}

En medio de la controversia generada por las nuevas guías de manejo del colesterol para reducir el riesgo cardiovascular (1), en las que los expertos afirman que "no hay evidencia que soporte el uso de un valor de colesterol LDL ni de colesterol no-HDL como meta de tratamiento", ni un valor de cLDL para iniciar intervención terapéutica ya que "en prevención primaria existe evidencia de beneficio del uso de medicamentos en el amplio rango de c-LDL desde 70 hasta $189 \mathrm{mg} / \mathrm{dL}$ ", surge la necesidad de establecer los valores "normales" de lípidos en cada país y me atrevería a decir en cada laboratorio clínico, a propósito del artículo publicado en este número de la revista (2).

¿Por qué todavía hay serias controversias alrededor de: a) los valores normales o meta de colesterol, b) los grupos de pacientes que se benefician del uso de medicamentos para bajarlo (o mejor de cuáles grupos no se benefician), c) la intervención nutricional adecuada? Porque, aunque está clara la relación directa entre colesterol y riesgo cardiovascular, ésta es una historia relativamente reciente y muchas de las conclusiones de las que han derivado las cambiantes recomendaciones de expertos, fueron el resultado de estudios que ahora sabemos, adolecían de imperfecciones $\mathrm{y}$ fallas.

Si revisamos la historia del colesterol, a pesar de varios estudios epidemiológicos, experimentales y algunos pocos de intervención, que sugerían una relación causal con la enfermedad ateroesclerótica, eran más los escépticos, y sólo hasta 1984 con la publicación del primer estudio aleatorizado doble ciego: The Coronary Primary Prevention Trial, que demostró una reducción significativa de eventos $\mathrm{CV}$ con la reducción de colesterol (con una resina fijadora de ácidos biliares), se citó a la primera Conferencia de Consenso (NIH) que publicó el primer consenso en JAMA (1985) sobre bajar el colesterol para prevenir ECV.
Merece la pena decir, que este consenso tuvo muchos y muy fuertes detractores, como los tuvieron las estatinas desde su aparición en el mercado en septiembre de 1987, hasta la publicación de los estudios más recientes y conocidos (4S, WOSCOPS, CARE, LIPID, AFCAPS/TexCAPS entre 1994 y 1998) que no dejan duda de lo que ahora ya tenemos por dogma $(3,4)$.

Entrando en el tema de los valores normales de colesterol, y hablando de historia, en los años 1940 y utilizando el patrón clásico de distribución normal y percentil 95, en Estados Unidos el 95\% de la población tenía un valor de colesterol sérico $<280 \mathrm{mg} / \mathrm{dL}$ (este fue el valor normal de referencia en USA hasta la publicación de las primeras guías de la ATP) (3). En 1955, y en vista de los primeros indicios de culpabilidad del colesterol, Ancel Keys inició el estudio epidemiológico tal vez más emblemático: El estudio de siete países, con el objetivo de ver la relación entre niveles de colesterol e ingesta de grasas en la dieta y muerte por ECV. Publicado en 1970, el estudio demostró que en todas las áreas geográficas incluidas, a mayor nivel de colesterol, mayor mortalidad coronaria: por ejemplo, en el este de Finlandia el colesterol promedio era $260 \mathrm{mg} / \mathrm{dL}$ y en Japón $160 \mathrm{mg} /$ $\mathrm{dL}$, cuya tasa de mortalidad en 10 años era $70 / 1000$ habitantes en el primero y 5/1000 en el segundo. Se cumplía para todos los países, que el riesgo de mortalidad era proporcional al nivel de colesterol en todo el rango de valores encontrados; pero aún no definieron un intervalo de referencia (3-5).

Los intervalos de referencia para los marcadores bioquímicos, son necesarios para establecer el diagnóstico y pronóstico de una enfermedad, la monitorización y seguimiento del parámetro medido, y aún más importante, para decidir cuándo hacer prevención, como es el caso de los niveles de lípidos y la enfermedad ateroesclerótica.
Ver artículo: página 29

Dra. María Inés Toro: InternistaEndocrinóloga Unidad de Endocrinología, Profesora de Endocrinología, Hospital Universitario de San Ignacio, Pontificia Universidad Javeriana. Bogotá, D.C. (Colombia)

E-mail: mitoro@hotmail.com 
Los métodos de medición son a menudo diferentes, y cada casa comercial provee intervalos de valores normales distintos, y como consecuencia la extrapolación de los valores entre laboratorios no es posible. Lo ideal es tener intervalos de referencia de la población, a la cual pertenecen los pacientes; ésta es la loable iniciativa del estudio que presenta en este número de la revista, el grupo de la Universidad de Antioquia: "Intervalos biológicos de referencia del perfil lipídico (2).

Estos intervalos varían con el género, la edad, la raza y muchos factores medioambientales (dieta, actividad física) y socioeconómicos, lo cual hace aún más difícil establecer "valores normales de referencia" con los que vamos a etiquetar a un paciente como sano, enfermo o en riesgo. Además, los rangos de referencia tienden a hacer pensar en límites definitivos que separan valores "buenos" de valores "malos", cuando en realidad lo que existe es un riesgo continuo que aumenta a medida que el valor del parámetro medido se aleja del valor óptimo e incluso, hablando de colesterol, el riesgo existe aun por debajo de este valor.

Hace aún más difícil la tarea de definir los parámetros de normalidad, la tarea previa de definir una población de sujetos sanos como población de referencia. Como ejemplos históricos, se han utilizado poblaciones que realmente no son representativas, como: estudiantes de medicina, empleados de instituciones de salud, donantes de sangre, entre otros.

Idealmente los valores de referencia se deben obtener de una población sana, que abarque todos los rangos de edad. El Instituto de Estándares para laboratorio clínico (CLSI), recomienda un tamaño mínimo de muestra de 120 sujetos para determinar intervalos de confianza de $90 \%$ para el percentil 95 de los límites de referencia (percentiles $2.5 \mathrm{y}$ 97.5) (6).

Aunque la Federación Internacional de Química Clínica (IFCC) recomienda el método directo para escoger la población: selección de sujetos con criterios de inclusión/ exclusión antes de tomar las muestras, es más utilizado el método indirecto, tal como se hizo en el presente estudio y como muy bien lo explican sus autores, se aplican los criterios de inclusión/exclusión y la división de la población por edad y género a posteriori a una base de datos existente en el laboratorio aplicando métodos estadísticos; este método es más barato, y muy útil para lograr el tamaño de la muestra necesario (7).

En lo referente al colesterol (y al perfil lipídico en general) se utilizan rangos óptimos (óptimos para la salud o que suponen un riesgo mínimo) y rangos terapéuticos, definidos por el ATP NCEP con base en muchos estudios y que establecieron el límite superior de colesterol total para tomar una decisión terapéutica, en $200 \mathrm{mg} / \mathrm{dL}$ (cLDL en $100 \mathrm{mg} / \mathrm{dL}$, triglicéridos en $150 \mathrm{mg} / \mathrm{dL}$ ) (8), y no el rango de referencia basado en la distribución normal de la población, que para un grupo de población sana de Estados Unidos, por ejemplo, estaría entre 139 y 273 $\mathrm{mg} / \mathrm{dL}$ para colesterol total (3).

Varias iniciativas recientes han logrado importantes avances para actualizar los intervalos de referencia de varios biomarcadores. Entre éstas, el Proyecto Nórdico de Intervalos de Referencia (9), el proyecto REALAB (10) y el CALIPER (11). En este último para dar un ejemplo, de una muestra de 12000 sujetos (aproximadamente 500 para cada grupo edad y género) los valores de referencia mostraron un patrón de cambio dinámico con la edad, con lo cual debieron dividir varios grupos etarios (diferentes a los predeterminados) para algunas variables como el colesterol total. En este estudio los triglicéridos aumentaron a partir de los 30 años de edad, más en hombres que en mujeres; el CLDL aumentó a partir de los 25 años y de nuevo más en hombres, y el colesterol total requirió seis grupos etarios sin diferencia entre géneros, con disminución en la adolescencia y aumento progresivo de los 20 a los 76 años (Tabla 1). En el estudio publicado en este número, por el contrario sí encontraron diferencias entre hombres y mujeres en el colesterol total y en las relaciones CT/CHDL y TGD/CHDL, y similar al estudio canadiense, los valores de triglicéridos fueron menores en los jóvenes que en adultos medios y mayores.

De estas iniciativas podemos prever que es necesario obtener intervalos de referencia biológicos propios, para géneros y grupos etarios, y muy probablemente por región dadas las diferencias culturales que implican diferentes hábitos alimentarios y de actividad física, que nos permitan interpretar mejor los resultados que nos reportan y sobre todo que nos permitan anticipar los mismos e iniciar medidas de prevención o de intervención cuando sea pertinente en nuestra población. 
Tabla 1. Intervalos de referencia en el CALIPER.

\begin{tabular}{|c|c|c|c|c|c|c|c|c|c|}
\hline & \multirow[b]{2}{*}{ Rang o edad } & \multicolumn{4}{|c|}{ Hombres } & \multicolumn{4}{|c|}{ Mujeres } \\
\hline & & Valor inf. & Valor sup. & Mediana & $\mathbf{n}$ & Valor inf. & Valor sup. & mediana & $\mathbf{n}$ \\
\hline \multirow[t]{3}{*}{ CTot al } & 2029 & 116 & 228 & 166 & 580 & 116 & 228 & 166 & 580 \\
\hline & 3039 & 147 & 266 & 185 & 900 & 147 & 266 & 185 & 900 \\
\hline & 4079 & 139 & 274 & 201 & 1710 & 139 & 274 & 201 & 1710 \\
\hline \multirow[t]{2}{*}{ CLD L } & 2549 & 62 & 189 & 124 & 480 & 50 & 178 & 104 & 540 \\
\hline & 5079 & 73 & 189 & 127 & 450 & 73 & 189 & 127 & 450 \\
\hline TGD-s & 3079 & 44 & 301 & 115 & 640 & 35 & 212 & 88 & 700 \\
\hline
\end{tabular}

\section{Referencias}

1. Circulation. 2014 Jun 24; 129 (25 Suppl 2): S145. 2013 ACC/AHA Guideline on the Treatment of Blood Cholesterol to Reduce Atherosclerotic Cardiovascular Risk in Adults http://circ.ahajournals.org/

2. Galvis Y, Barona J, Cardona JA. Intervalos biológicos de referencia del perfil lipídico. Acta Med Colomb 2016; 41: 29-35.

3. Steinberg D. J of Lipid Res. 2004. Thematic review series: The Pathogenesis of Atherosclerosis. An interpretive history of the cholesterol controversy: parts I, II, III , IV \& V.

4. Akira E. Proc Jpn Acad Ser B. Phys Biol Sci. 2010 May 11, 86 (5): 484-493. A historical perspective on the Discovery of statins.

5. Scientific American. http://blogs.scientificamerican.com/ guestblog/cholesterolconfusionandwhyweshouldrethinkoura pproachtostatintherapy/.

6. Clinical Laboratory Standards Institute. Defining, Estab- lishing, and Verifying Reference Intervals in the Clinical Laboratory; EP28A3C: Approved Guideline-Third Edition. http:// shop.clsi.org/c.1253739//Sample_pdf/EP28A3C_sample.pdf.

7. JIFCC. 2008, 19 (2): 111 Gary L Horowitz Reference Intervals. Practical aspects.

8. CMAJ. 28, vol. 169 no. 9. www.cmaj.ca/cgi/content/ full/169/9/921/DC1.

9. The Nordic Reference Interval Project 2000 Recommended reference intervals for 25 common biochemical properties. Scand J of Clin and Lb Invest. 2004; 64 (4): 271-284.

10. The REALAB Project: A new Method for the formulation of reference intervals based on current data. Clin Chem. 2005; 51 (7): 1232-1240.

11. Biochemical marker reference values across pediatric adult and geriatric ages: establishment of robust pediatric and adult reference intervals on the basis of the Canadian Health Measures Survey. Clin Chem. 2015; 61 (8): 1049-1062. 